\title{
Upregulated Monocyte Expression of Plin2 Depends Upon Proteasome Impairment and is Associated with Early Atherosclerosis in Obese Children
}

\section{Eugenia Pisano}

Department of Cardiovascular Science, Fondazione Policlinico Universitario A. Gemelli, IRCCS, Rome, Italy

\section{Lucia Pacifico}

Policlinico Umberto I Hospital, Sapienza University of Rome, Viale Regina Elena 324, 00161 Rome, Italy

\section{Francesco Massimo Perla}

Policlinico Umberto I Hospital, Sapienza University of Rome, Viale Regina Elena 324, 00161 Rome, Italy

\section{Giovanna Liuzzo}

Department of Cardiovascular Science, Fondazione Policlinico Universitario A. Gemelli, IRCCS, Rome, Italy

\section{Claudio Chiesa}

Institute of Translational Pharmacology, National Research Council, Via del Fosso del Cavaliere, 100, 00133-Rome, Italy

\section{Michela Lavorato}

Policlinico Umberto I Hospital, Sapienza University of Rome, Viale Regina Elena 324, 00161 Rome, Italy

\section{Geltrude Mingrone}

Department of Internal Medicine, Fondazione Policlinico Universitario A. Gemelli, IRCCS, Rome Italy; Department of Diabetes, King's College London, United Kingdom.

\section{Marta Fabrizi}

Research Area for Multifactorial Diseases and Complex Phenotypes, Obesity and Diabetes, Bambino Gesù Children's Hospital, IRCCS, Rome, Italy.

\section{Danilo Fintini}

Endocrinology Unit, Bambino Gesù Children's Hospital, IRCCS, Palidoro, Rome, Italy

\section{Anna Severino ( $\square$ anna.severino1@unicatt.it)}

Research Area for Multifactorial Diseases and Complex Phenotypes, Obesity and Diabetes, Bambino Gesù Children's Hospital, IRCCS, Rome, Italy; Catholic University of Sacred Heart, Rome, Italy https://orcid.org/0000-0002-6276-7747

\section{Melania Manco}

Research Area for Multifactorial Diseases and Complex Phenotypes, Obesity and Diabetes, Bambino Gesù Children's Hospital, IRCCS, Rome, Italy. 
Research

Keywords: atherosclerosis, cardiovascular disease, children obesity, perilipins_Plin2, proteasome, CMA, personalized medicine.

Posted Date: September 8th, 2020

DOI: https://doi.org/10.21203/rs.3.rs-70773/v1

License: (c) (i) This work is licensed under a Creative Commons Attribution 4.0 International License.

Read Full License 


\section{Abstract}

Background: Perilipin (Plin) 2 regulates intracellular lipid metabolism in macrophages, thus playing a role in atherosclerosis. Aim of the study was to evaluate whether dysregulation of Plin2 is involved in the onset of the early atherosclerosis seen in children with obesity and to rule out mechanisms of dysregulation.

Methods: We enrolled 63 children with overweight/obesity and 21 age- and sex-matched normal-weight controls; we evaluated carotid intima media thickness (cIMT). We determined mRNA expression of Plin2 and proteasome subunits (PSMD3, PSMC4) by RealTime PCR and protein expression of Plin2, LAMP2A and Hsc70 by western blot analysis. We performed transient LAMP2A downregulation by siRNA. We quantified intracellular lipids in monocytes by Nile Red staining and flow cytometry analysis.

Results: Levels of Plin2 protein were significantly higher in obese children than in normal-weight controls and correlated with cIMT in children with obesity after adjusting for confounders. Accordingly, monocytes of children with obesity showed a higher intracellular amount of lipids compared to normal weight children. mRNA expression of the regulatory subunits PSMC4 and PSMD3 and proteasome activity were lower in children with obesity than in controls while expression of LAMP2A and Hsc70 proteins, belonging to chaperone mediated autophagy (CMA) pathway, was not different indicating that Plin2 dysregulation in monocytes was not CMA-mediated but likely due to an impairment of proteasome efficiency.

Conclusion: Plin2 was overexpressed in monocytes of children with obesity likely owing to reduced protein degradation by the proteasome. Overexpression of Plin2 could contribute to the early onset of atherosclerosis in these children.

\section{Background}

Childhood obesity represents one of the foremost global health threats [1]. Obesity accelerates the atherogenic process that starts in childhood, progresses throughout the lifespan and results in overt CVD in adult age [2].

In children and adolescents, the extent of early signs of atherosclerosis is directly associated with the severity of obesity comorbidities, i.e. dyslipidemia and high blood pressure [3]. Atherosclerosis begins as fatty streaks in the intima of arteries. Autopsies of healthy young individuals documented that aortic surface involvement with fatty streaks occurs at early ages and the extension of such involvement correlates with ante-mortem total and low lipoprotein density (LDL) cholesterol levels [4]. cIMT provides a direct estimate of atherosclerosis in children and is a convenient risk factor of the overall CVD in later adulthood [5].

The differentiation of macrophages into foam cells, caused by massive accumulation of lipid droplets (LDs), is key factor in the fatty streak onset. LDs are highly regulated organelles, composed by an internal lipid core surrounded by a phospholipid monolayer and numerous proteins with regulatory functions that 
belong to the PAT family. The family encloses five members, known as Perilipins (Plins) 1 to 5 , characterized with respect to their structure, function and ectopic expression [6]. Plin2 is expressed in macrophages and it is over-expressed in human carotid lesions [7]. In atherosclerotic lesions, thin fibrous cap, holding Plin2-positive macrophages, correlated with intraplaque hemorrhage and high-risk carotid lesions [8]. Conversely, macrophages from Plin2-deficient mice displayed a reduced ability to become foam cells and these mice were protected against atherosclerosis at the aortic sinus [9]. Plin2 modulates the net balance between cellular intake and efflux of fat, enhancing the lipogenesis and down-regulating the lipolysis [10-12].

The expression of Plin2 in monocytes and macrophages is transcriptionally regulated by oxLDL, acLDL [13] and by peroxisome proliferator-activated nuclear receptor synthetic agonists. The lipopolysaccharide (LPS) also up regulates Plin2 expression [14].

Plin2 expression is post-translationally regulated by CMA and proteolytic degradation via the ubiquitin/proteasome pathway. The CMA-mediated degradation of the protein has been robustly demonstrated to occur in the liver [15]. Whether it regulates expression of Plin2 protein in monocytes is still unknown. CMA renders LDs accessible to lipase and facilitates mobilization of intracellular lipid stores. The molecular chaperone Hsc70 interacts directly with Plin2 on LDs and drives it to lysosomeassociated membrane protein 2A (LAMP2A), the rate-limiting component of the pathway [16-18]. While a physiological activator of CMA is nutrient deprivation, dietary lipids and aging have an inhibitory effect, reducing the stability of LAMP2A on the lysosome membrane [19]. A fundamental step for Plin2 delivery

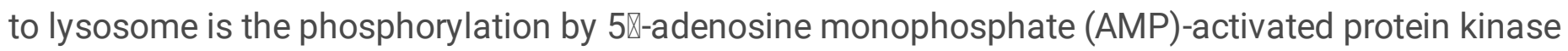
(AMPK) [20], whose activity is impaired in patients with obesity [21].

Plin2 proteasome dependent proteolysis is induced by the absence of an external lipid source in cultured cells and is accompanied by lipid store reduction. Environmental factors and signaling molecules that regulate ubiquitination and proteasome-induced degradation of Plin2 are unknown, but it seems that oleate and lipoproteins reduce protein degradation [22-24]. Efficiency of the proteasome-dependent proteolysis is reduced in murine models of accelerated aging and in human subjects with obesity [2527].

Aims of the present study were: 1) to investigate whether expression of Plin2 is dysregulated in monocytes of children with obesity as compared to normal weight controls and associated with early onset atherosclerosis, as estimated by measurement of the cIMT;2) to rule out mechanisms of Plin2 dysregulation associated with obesity.

\section{Materials And Method}

\section{Study subjects}

This cross-sectional study included 63 children with overweight (ow) or obesity and 21 normal-weight age- and sex-matched controls consecutively recruited at the outpatient "Hepatology, Lipid and Nutrition" 
Clinics of the Department of Pediatrics, Sapienza University of Rome, Italy, between March 2016 and December 2017. Healthy normal-weight controls were students drawn from randomly selected schools in the Rome area and invited to volunteer.

Study participants had complete physical examination, as reported in detail elsewhere [28-30]. Pubertal development was staged according to Tanner [31]. The degree of obesity was quantified using the Cole's least mean-square method, which normalizes the skewed distribution of body mass index (BMI) and expresses BMI as standard deviation (SD) score [32].

Inclusion criteria were age between 7 and 18 years-old, Caucasian ethnicity, no alcohol or smoking habit, no consumption of any drug, no chronic, endocrine/genetic or acute disease except for obesity and associated comorbidities.

The study protocol conformed to the ethical guidelines of the 1975 Helsinki Declaration as reflected in the approval of the Ethics Committee of Policlinico Umberto I Hospital, Rome, Italy. Written informed consent was obtained from children's parents/guardians.

\section{Laboratory Measurements}

Glucose, insulin, total cholesterol, low density lipoprotein-cholesterol (LDL-C), high density lipoproteincholesterol (HDL-C), triglycerides, alanine aminotransferase (ALT), aspartate aminotransferase (AST), and gamma-glutamyl transferase were assayed on children's blood samples after overnight fast. Insulin resistance was calculated by the homeostasis model assessment of insulin resistance (HOMA-IR) [33]. All analyses were performed on COBAS 6000 (Roche Diagnostics, Risch-Rotkreuz, Switzerland).

E-Selectin (MyBiosource, San Diego, USA) and LPS (HycultBiotech, Plymouth Meeting, PA, USA) measurement were performed by commercial ELISA kit following the manufacturer's instructions.

\section{Case definitions}

Ow and obesity were defined according to age- and gender-specific cut-off points of BMI defined by the International Obesity Task Force criteria as proposed by Cole et al. [32]. Elevated blood pressure (BP) was defined as systolic or diastolic percentile for age, gender, and height [34]. Impaired fasting glucose was defined as glucose $\geq 5.6 \mathrm{mmol} / \mathrm{L}$. High triglycerides, and low HDL-C were defined using the cut-off proposed by Cook et al. [35]. Insulin resistance was defined by the 90th percentile of HOMA-IR for age and gender in the Italian population [36].

\section{Carotid ultrasonography}

Carotid ultrasonography was performed in all patients by a single experienced radiologist, who was blind to participants' details. cIMT was measured as previously described $[29,30]$. In brief, longitudinal ultrasonographic scans of the carotid artery included the evaluation of the right and left common carotid arteries near the bifurcation during end-diastole. We measured four values on each side, and the maximum and mean cIMT were calculated. The coefficient of variation was less than $3 \%$. 


\section{Monocyte isolation}

Peripheral blood mononuclear cells (PBMCs) were isolated from EDTA whole blood samples through standard gradient centrifugation over Ficoll-Hypaque (GE Healthcare Bio-Sciences, Piscataway, New Jersey). Monocytes were purified by Pan Monocytes Isolation Kit (Miltenyi) through indirect magnetic labelling of PBMCs and cell population purity was assessed by flow cytometry analysis. Monocyte pellets were stored at $-80^{\circ} \mathrm{C}$ for further analysis.

\section{Western Blot Analysis}

Monocytes were lysed in RIPA buffer containing a cocktail of protease inhibitors. Protein content was determined using Bradford Protein Assay (Bio-Rad). Protein lysates were separated on 8\% SDS-PAGE and transferred on polyvinylidene fluoride membrane. Membranes were probed overnight with the following monoclonal antibodies: Plin2 AP125 (LifeSpanBioSciences,Inc), LAMP2A H4B4 (Abcam), Hsc70 13D3 (Abcam) and $\beta$-actin C-4 (Santa Cruz). Detection and analysis were performed with the ChemiDoc XRS system with Image Lab 5.0 software (Bio-Rad). All results were normalized with $\beta$-actin and expressed as ratio.

\section{Nile Red staining}

PBMCs from children with overweight or obesity and normal weight controls, were stained for 45 min at $37^{\circ} \mathrm{C}$ in $5 \%$ CO2 with Nile Red $(100 \mathrm{ng} / \mathrm{mL}$ ) (Sigma-Aldrich). Then, PBMCs were incubated for $15 \mathrm{~min}$ with anti-CD14-FITC (Beckman Coulter) to identify monocyte population. Flow cytometry analysis was conducted with FC 500 (Beckman Coulter) and the data analyzed with Kaluza software (Beckman Coulter).

\section{RNA extraction and Quantitative Real Time PCR Analysis}

Total RNA was extracted from purified monocytes using the RNeasy Plus Mini Kit (Qiagen GmbH, Hilden, Germany) according to the indications provided by the company. A small aliquot of total RNA obtained was subjected to qualitative and quantitative control by nanodrop analysis (Thermo Fisher Scientific, Waltham, Massachusetts). Total RNA was reverse-transcribed into cDNA by using iScript RT (Bio-Rad, Hercules, California). SYBR Green gene expression assays were performed in triplicate using the iQ SYBR Green Supermix (Bio-Rad, Hercules, California) and the iQ5 Multicolor Real-Time PCR Detection System (Bio-Rad, Hercules, California). The primer pairs used for qPCR in this study were listed in Table 2. 
Table 2

Primer used in this study

\begin{tabular}{|ll|}
\hline Primer name & Primer sequence $\mathbf{5}^{\prime}$ to $\mathbf{3}^{\prime}$ \\
\hline Plin2-F & AAAGCCAACAGACCATTTC \\
\hline Plin2-R & TTATCCTGAGCATCCTGAAT \\
\hline PSMD3-F & ACCTCTACTACACAGGGCGA \\
\hline PSMD3-R & AACAGCTCCACCACGATGAG \\
\hline PSMC4-F & AGGTGCAGGAGGAATACATCAA \\
\hline PSMC4-R & AATAGTTGGAGCCTGTGGTAGA \\
\hline PSMA6-F & GTGAGTTCGGCATGCAAGAG \\
\hline PSMA6-R & GCCTTAAAAGCATATTCTACTTGGT \\
\hline PSMB7-F & GGAGCAGATACAAGAGCAACTG \\
\hline PSMA7-R & GTCCCAGCACCACAACAATAAA \\
\hline B2M-F & AGGACTGGTCTTTCTATCTCTTGT \\
\hline B2M-R & ACCTCCATGATGCTGCTTACA \\
\hline
\end{tabular}

Expression levels were normalized to $\beta 2$ Microglobulin ( $\beta 2 \mathrm{M})$. Relative gene expression was calculated using the 2- $\Delta \Delta \mathrm{CT}$ (comparative threshold) method.

\section{Primary monocyte culture}

Monocytes were maintained in RPMI-1640 containing fetal bovine serum (10\%v/v), penicillin/streptomycin $(50 \mathrm{U} / \mathrm{ml} / 50 \mathrm{ug} / \mathrm{ml})$, and L-glutamine $(2 \mathrm{mM})$. Monocytes were seeded at a density of $1 \times 10^{6}$ cells $/ \mathrm{ml}$ and treated with Compound C $10 \mu \mathrm{M}$ and MG132 $10 \mu \mathrm{M}$ (specific inhibitors of AMPK and the proteasome, respectively) all from Sigma Aldrich.

\section{LAMP2A silencing}

Isolated monocytes were transfected with $100 \mathrm{nM}$ gene specific siRNA duplexes for LAMP2A and a corresponding quantity of Scrambled duplex (OriGene) using TransIT-TKO cationic lipids method (Mirus). Cells were harvested $48 \mathrm{~h}$ after transfection, without medium replenishment. LAMP2A and PLIN2 proteins were quantified as described.

\section{Proteasome activity}

Monocyte pellets were lysed in 0.5\% NP-40 and protein content was determined using Bradford Protein Assay (Bio-Rad). Proteasome activity was measured by Fluorimetric ( $E x / E m=350 / 440 \mathrm{~nm}$ ) Proteasome Activity Assay kit (Abcam) following manufacturer's instructions. Proteasome activity was calculated as follow: RFU/min/mg total proteins. 


\section{Statistical Analysis}

Continuous variables that were normally distributed, as assessed by Shapiro Wilk test or KolmogorovSmirnov test, were described as mean \pm Standard Error of the Mean (SEM) or standard deviation (SD). Data that did not follow a normal distribution were presented as median (interquartile range) and were analyzed by using nonparametric test. In particular unpaired $t$ test (for continuous variables that were normally distributed), Mann-Whitney $\mathrm{U}$ test (for nonparametric variables) and chi-square test (for categorical variables) were used as appropriate. One-way ANOVA for repeated measures, with Bonferroni correction, was used for multiple comparisons. The nonparametric Spearman rank test or the parametric Pearson test were used for correlation analysis. The strength of relations was expressed as coefficient and $p$ value. To ascertain whether monocyte Plin2 expression level was independently associated with CIMT, multivariable linear-regression was run after adjusting for age, sex, systolic blood pressure, diastolic blood pressure, BMI z-score, hyperlipidemia, HOMA-index, LPS and soluble E-selectin level. A $p$ value < 0.05 was considered statistically significant. Statistical analyses were performed with GraphPad Prism version 5.00 for Windows (GraphPad Software, La Jolla, San Diego CA) and with SPSS software v22.0 (IBM Corporation, Armonk, New York).

\section{Results}

\section{Plin2 was upregulated in monocytes of children with ow/obesity as compared to normal-weight controls.}

The two groups were not different with respect to gender distribution, age and Tanner stage, while ow/obese children had significantly higher fasting glucose $(p=0.0007)$, insulin ( $p<0.0001)$, HOMA-IR $(p<0.0001)$, cIMT $(p=0.04)$, and lower HDL-C $(p=0.01)$. Table 1 shows clinical, laboratory and carotid ultrasound characteristics of the study population.

Plin2 protein level was significantly higher in monocytes of children with ow/obesity as compared to controls [66.6 (3.453) vs. 45.12 (4.457); $p=0.001$ ] (Fig. 1A, Fig. 1B). mRNA level of Plin2 was not different in the two groups but tended to be lower in children with ow/obesity (Fig. 1C). These data suggested that overexpression of Plin2 is not due to any transcriptional dysregulation.

Accordingly, monocytes from children with ow/obesity showed a significant higher amount of intracellular lipids with respect to normal weight subjects [3.602 (0.2457) vs $1.652(0.055) ; p<0.0001]$ (Fig. 1D). 
Table 1

Clinical and laboratory characteristics of the subjects.

Data are expressed as n (\%), mean and SD (age) or median (interquartile ranges).

\begin{tabular}{|c|c|c|c|}
\hline & $\begin{array}{l}\text { Lean } \\
(n=21)\end{array}$ & $\begin{array}{l}\text { Overweight/Obese } \\
(n=63)\end{array}$ & ${ }^{*} p$-value \\
\hline \multicolumn{4}{|l|}{ Clinical characteristics } \\
\hline Age (years) & $12.2(0.63)$ & $11.9(0.28)$ & 0.64 \\
\hline Male sex (\%) & $10(47.6)$ & $38(60.3)$ & 0.90 \\
\hline Height (cm) & $154.5(139-62.1)$ & $157(147.8-165)$ & 0.22 \\
\hline Weight (Kg) & $45.5(31.6-53.5)$ & $62(51-79.1)$ & $<0.0001$ \\
\hline Body Mass Index (Kg/m2) & $19.5(17.6-21)$ & $25.8(23.3-29.1)$ & $<0.0001$ \\
\hline Weight z-score & $0.2(-0.5-0.84)$ & $2.3(1.96-2.7)$ & $<0.0001$ \\
\hline Systolic blood pressure (mmHg) & $110(105-120)$ & $115(110-120)$ & 0.07 \\
\hline Diastolic blood pressure (mmHg) & $65(62.5-70)$ & $70(65-75)$ & 0.02 \\
\hline Tanner Stage & & & 0.9815 \\
\hline T1 (\%) & $1(0.04)$ & $2(0.03)$ & \\
\hline T2 (\%) & $3(0.14)$ & $16(0.2)$ & \\
\hline T3 (\%) & $10(0.47)$ & $20(0.31)$ & \\
\hline T4 (\%) & $2(0.09)$ & $12(0.19)$ & \\
\hline T5 (\%) & $5(0.23)$ & $13(0.2)$ & \\
\hline \multicolumn{4}{|l|}{ Laboratory data } \\
\hline Triglycerides (mg/dL) & $66(50.2-93.7)$ & $78(58-125)$ & 0.06 \\
\hline Total-Cholesterol (mg/dL) & $179(150-201)$ & $164(139.5-187.5)$ & 0.15 \\
\hline HDL-Cholesterol (mg/dL) & $58.5(48.2-73.2)$ & $51(41.5-58)$ & 0.01 \\
\hline LDL-Cholesterol (mg/dL) & $109(72-131)$ & $92(75-113.5)$ & 0.40 \\
\hline AST (IU/L) & $22.5(15.7-23)$ & $21(18-28)$ & 0.40 \\
\hline ALT (IU/L) & $14(11.2-19.2)$ & $18.5(14-32)$ & 0.006 \\
\hline Y-GT (IU/L) & $10(9-13)$ & $13(10.2-18.7)$ & 0.02 \\
\hline Fasting Glucose (mg/dL) & $76(74-81)$ & $83(77-88)$ & 0.0007 \\
\hline Fasting Insulin ( $\mu \mathrm{U} / \mathrm{mL})$ & $8.8(5.5-10.5)$ & $16.6(12.8-23.3)$ & $<0.0001$ \\
\hline
\end{tabular}


Instrumental data

clntima Media Thickness (mm)

$0.37(0.34-0.45)$

$0.42(0.4-0.43)$

0.04

*Mann-Whitney $U$ test for continuous variables or $\chi 2$ test for categorical variables. LDL-cholesterol low-density lipoprotein cholesterol, HDL-cholesterol high-density lipoprotein cholesterol, ALT alanine aminotransferase, AST aspartate aminotransferase, $\mathrm{Y}$-GT gamma-glutamyl transferase, HOMA-IR homeostasis model assessment for insulin resistance, $\mathrm{HbA1c}$ glycated hemoglobin.

\section{Correlation of monocyte Plin2 expression, blood lipids and atherosclerosis in children with obesity.}

Expression of Plin2 in monocytes of children with obesity was significantly correlated with cIMT $(r=0.39$; $p=0.01$ ) (Fig. 2A), while it was not in controls. Multivariable linear-regression analysis showed a significant correlation between expression level of Plin2 in monocytes of children with obesity and cIMT after adjusting for a number of variables known to affect $\operatorname{cIMT}(\beta$-coeff $=0.39 ; p=0.02)$. The expression of Plin2 was not different between children with and without obesity, high total cholesterol, high LDL cholesterol, high triglycerides, or low HDL cholesterol, respectively (data not reported). Levels of Plin2 and $\mathrm{HDL}$ were significantly associated $(\mathrm{r}=0.35 p=0.03)$ in children with obesity when cases with overweight were excluded from the analysis (Fig. 2B).

\section{CMA regulates Plin2 protein level in human primary monocytes.}

To assess the role of CMA in the regulation of Plin2 in human monocytes, cells from healthy normal weight donors were transfected with LAMP2A targeting small interfering RNA (siRNA).

LAMP2A protein level was significantly reduced by 1.5 fold in comparison to control after $48 \mathrm{~h}(p=0.03)$. In parallel, a 3.2 fold increase of Plin2 protein level was observed ( $p=0.03$ ) (Fig. $3 \mathrm{~A}$ ) confirming that CMA regulates Plin2 expression also in human monocytes.

\section{Inhibition of AMPK and proteasome results in increased expression of Plin2 in human primary monocytes.}

Primary monocytes isolated from human donors (T0) and cultivated in the absence of an external lipid source in culture medium (control), showed a rapid degradation of Plin2 due to post-translational regulation of the protein. Plin2 protein expression was increased in monocytes treated with Compound $\mathrm{C}$ 
and MG132 (specific inhibitors of AMPK and the proteasome, respectively) as compared to not treated control cells [28.3 (3) and 45.02 (2.8) vs $2.5(0.65) ; p=0.003, \mathrm{p}=0.0004$ and $p=0.0012$ respectively]. The inhibition of both mechanisms had an additive effect on the increased expression of Plin2 as compared to the control [155.0 (12.8) vs $2.6(0.65) ; p=0.003$ ] (ANOVA for trend $p<0.0001)$ (Fig. 3B). Thus, both mechanisms are involved in the post-translational regulation of Plin2 in monocytes.

\section{Upregulation of Plin2 in monocytes of children with ow/obesity is not dependent on CMA impairment.}

To rule out whether degradation of Plin2 by CMA is impaired in ow/obese patients, expression of Hsc70 (data not shown) and LAMP2A protein was analyzed, but no significant difference was found between children with ow/obesity and controls. Nevertheless, while levels of Plin2 and LAMP2A were inversely correlated in normal-weight subjects $(r=-0.75 ; p=0.0004)$ they were not in children with ow/obesity (Fig. 4). Since $L A M P 2 A$ is the rate-limiting factor of CMA, these data suggest that Plin2 overexpression in monocytes of ow/obese children is not due to an impairment of CMA.

\section{Expression level of proteasome regulatory subunits and proteasome activity are lower in monocytes of children with ow/obesity.}

We evaluated mRNA expression of proteasome regulatory (PSMC4, PSMD3) and catalytic subunits (PSMA6, PSMB7) in monocytes of normal-weight and children with ow/obesity. Controls and cases with ow/obesity showed a significant difference in mRNA expression of PSMC4 and PSMD3 [155.1 (16.8) vs 105.8 (8.6); $p=0.006$; and 11.7 (1.5) vs $8.6(0.66) ; p=0.03$ ] but not in mRNA expression of catalytic proteasome subunits (data not shown) (Fig. 5A). In children with ow/obesity, mRNA levels of PSMC4 and PSMD3 were inversely correlated with protein expression of Plin2 $(r=-0.42, p=0.003$ and $r=-0.32, p=0.02$, respectively) while expression of catalytic subunits was not (data not shown) (Fig. 5B). In addition, proteolytic activity of proteasome was lower in ow/obese patients although not significantly (Fig. 5C). Together these results suggest that an impaired proteasome efficiency may lead to the decreased degradation of Plin2.

\section{Discussion}

We found that Plin2 is significantly overexpressed in monocytes of children with ow/obesity as compared with controls, likely owing to an impairment of the proteasome dependent degradative pathway. Likewise, levels of Plin2 protein were significantly correlated with cIMT, a surrogate of atherosclerosis. This is the first report in individuals with obesity. 
The role of Plin2 has been investigated in relation to two conditions that are strongly associated, i.e. the atherosclerosis and the non-alcoholic fatty liver disease (NAFLD) [37]. It is likely that the dysregulation of the protein both in macrophages and in liver is pivotal to explain the co-occurrence of the various obesity co-morbidities, in particular atherosclerosis and NAFLD.

\section{Over-expression of Plin2 is associated with early atherosclerosis.}

Human and murine models of atherosclerosis support strongly the pivotal role of Plin2 in the formation of foam cells. Very recently, we found that upregulation of Plin2 is associated with neoatherogenesis in patients with restenosis after drug-eluting stent implantation [38].

Our study supports the role of Plin2 in the pathogenesis of human early atherosclerosis given the correlation between Plin2 protein level and cIMT.

Dyslipidemia of people with obesity is mainly characterized by reduced HDL-C. In our series, levels of Plin2 expression were correlated with HDL-C but not with LDL-C, triglycerides and ApoB. A correlation trend was observed between Plin2 protein expression and ApoA1 levels, too. These data could suggest a compensative response, favoring reverse cholesterol transport in monocytes/macrophages of children with obesity who have increased intracellular levels of Plin2.

Such as Plin2 has been proposed as a therapeutic target to prevent atherosclerosis. In double ApoE and Plin2 knockout mouse model, the increase of plasma ApoA1 enhanced athero-protection and influenced positively remodeling of the lesion and reversal of the cholesterol transport [39].

\section{Impairment of Plin2 degradation in children with obesity.}

Inside the mechanism, our findings demonstrate that the degradation of Plin2 is impaired in children with obesity and causes the upregulation of the protein. On the contrary, there is no transcriptional dysregulation, since the mRNA expression was not different in normal-weight and children with obesity. We investigated first CMA in monocytes of healthy donors demonstrating that this pathway regulates Plin2 protein level in myeloid cells similarly to what it does in the liver [15]. In fact, disruption of CMA downstream, by LAMP2A silencing, and upstream, by AMPK signaling inhibition, led to a significant increase of Plin2 protein level. Thus, both the CMA and the proteasome degradation pathways regulate Plin2 in monocytes of healthy individuals.

Then, we evaluated LAMP2A and Hsc70 protein expression in children normal-weight and with obesity but we did not find a significant difference between the two groups; while there was a significant inverse correlation between Plin2 and LAMP2A in normal-weight individuals but not in those with obesity. 
After having ruled out the role of deregulated CMA as cause of Plin2 overexpression in children with obesity, we tested the hypothesis of an impairment of the proteasome degradation pathway.

We analyzed mRNA expression of the proteasome regulatory subunits PSMD3 and PSMC4 and the catalytic subunits PSMA6 and PSMB7. We found that expression of regulatory subunits was significantly downregulated in obese children in keeping with previous observation in obese adults [40] and that levels of Plin2 protein level and mRNA expression of proteasome regulatory subunits were inversely correlated. In addition, the proteasome activity was lower in children with ow/obesity (Fig. 6).

Free fatty acids, insulin and oxidative stress can all affect proteasome functionality in liver and adipose tissue of individuals with obesity, worsening insulin resistance [41].

In our setting, down-regulation of the proteasome could favor Plin2 accumulation in circulating monocytes of children with obesity contributing to early onset of the atherogenic process.

\section{Study Limitation}

We are aware of caveats that require further investigation. The present study was a cross-sectional investigation of less controls and more cases. Indeed, it was not easy to enroll and study with blood sample school -age children and adolescents who had to skip school. Investigation was also limited by the amount of stored monocyte lysates. We provided evidence on the association between protein overexpression and cIMT but longitudinal data are warranted to demonstrate that Plin2 is a marker of increased risk.

\section{Conclusions}

Our work demonstrated, for the first time, Plin2 overexpression in monocytes of children with ow/obesity and the significant association to the carotid intima media thickness, an early marker of atherosclerosis. Plin2 was likely upregulated owing to impaired degradative processes rather than to transcriptional dysregulation. Since it is widely accepted that proteasome efficiency is reduced in obesity and aging, its impairment is likely to accelerate in children with obesity the atherosclerotic process that occurs physiologically with aging, hence anticipating the onset of symptomatic disease.

\section{Abbreviations}

CVD- Cardiovascular Disease

cIMT- carotid Intima-Media Thickness

LD- Lipid Droplet

CMA- Chaperone Mediated Autophagy 
NAFLD- Non-Alcoholic Fatty Liver Disease

LPS- Lipopolysaccharide

PBMC- Peripheral Blood Mononuclear Cell

AMPK 5囚-Adenosine Monophosphate (AMP)-activated protein Kinase

\section{Declarations}

\section{Ethics approval and consent to participate:}

This study was approved by the Ethics Committee of Policlinico Umberto I Hospital, Rome, Italy. Written informed consent was obtained from children's parents/guardians.

\section{Consent for publication:}

Not applicable

\section{Availability of data and materials:}

All data generated or analyzed during this study are included in this published article. Any dataset used and/or analyzed during the current study are available from the corresponding author on reasonable request.

\section{Competing interests:}

No potential conflicts of interest relevant to this article were reported.

\section{Funding:}

The study was funded by the Italian Ministry of Health under the grant Ricerca Corrente RC201780X003315. The funder had no role in the design of the study, writing and submitting the manuscript.

\section{Authors' contributions:}

A.S. and M.M. contributed to the experimental design, data analysis, and writing of the manuscript. L.P. M.P. and M.L. contributed to children enrolment. E.P and M.F. contributed to experiments. G.L. C.C. and G.M. contributed to the experimental design, data analysis, and writing of the manuscript. A.S. is the 
guarantor of this work and, as such, had full access to all the data in the study and takes responsibility for the integrity of the data and the accuracy of the data analysis.

\section{Acknowledgements:}

Not applicable.

\section{References}

1. Steinberger J, Daniels SR, Eckel RH, Hayman L, Lustig RH, et al. Progress and challenges in metabolic syndrome in children and adolescents: a scientific statement from the American Heart Association Atherosclerosis, Hypertension, and Obesity in the Young Committee of the Council on Cardiovascular Disease in the Young; Council on Cardiovascular Nursing; and Council on Nutrition, Physical Activity, and Metabolism. Circulation. 2009;119(4):628-747.

2. Berenson GS. Cardiovascular risk begins in childhood: a time for action. The American Journal of Preventive Medicine. 2009;37(1):1-2.

3. Olson M, Chambers M, Shaibi G. Pediatric markers of adult cardiovascular disease. Curr Pediatr Rev. 2017;13(4):255-9.

4. Newman WP, Freedman DS, Voors AW, Gard PD, Srinivasan SR, et al. Relation of serum lipoprotein levels and systolic blood pressure to early atherosclerosis. The Bogalusa Heart Study. N Engl J Med. 1986;314:138-44.

5. Urbina EM, Williams RV, Alpert BS, Collins RT, Daniels SR, et al. Noninvasive assessment of subclinical atherosclerosis in children and adolescents: recommendations for standard assessment for clinical research: a scientific statement from the American Heart Association. Hypertension. 2009;54(5):919-50.

6. Kimmel AR, Brasaemle DL, McAndrews-Hill M, Sztalryd C, Londos C. Adoption of PERILIPIN as a unifying nomenclature for the mammalian PAT-family of intracellular lipid storage droplet proteins. $J$ Lipid Res. 2010;51:468-71.

7. Collot-Teixeira S, Barbatis C, Bultelle F, Koutouzis M, Pasterkamp G, et al. CD36 is significantly correlated with adipophilin in human carotid lesions and inversely correlated with plasma ApoAl. $J$ Biomed Biotechnol. Volume 2008, 813236.

8. Hao H, lihara K, Ishibashi-Ueda H, Saito F, Hirota S. Correlation of thin fibrous cap possessing adipophilin-positive macrophages and intraplaque hemorrhage with high clinical risk for carotid endarterectomy. J Neurosurg. 2011;114(4):1080-7.

9. Paul A, Chang BH, Li L, Yechoor VK, Chan L. Deficiency of adipose differentiation-related protein impairs foam cell formation and protects against atherosclerosis. Circ Res. 2008;102:1492-501.

10. Larigauderie G, Furman C, Jaye M, Lasselin C, Copin C, et al. Adipophilin enhances lipid accumulation and prevents lipid efflux from THP-1 macrophages: potential role in atherogenesis. 
Arterioscler Thromb Vasc Biol. 2004;24(3):504-10.

11. Larigauderie G, Cuaz-Pérolin C, Younes AB, Furman C, Lasselin C, et al. Adipophilin increases triglyceyde storage in human macrophages by stimulation of biosynthesis and inhibition of betaoxidation. FEBS. 2006;273(15):3498-510.

12. Listenberger LL, Ostermeyer-Fay AG, Goldberg EB, Brown WJ, Brown DA. Adipocyte differentiationrelated protein reduces the lipid droplet association of adipose triglyceride lipase and slows triacylglycerol turnover. J Lipid Res. 2007;48(12):2751-61.

13. Varela LM, López S, Ortega-Gómez A, Bermúdez B, Buers I, et al. Postprandial triglyceride-rich lipoproteins regulate perilipin-2 and perilipin-3 lipid droplet-associated proteins in macrophages. $J$ Nutr Biochem. 2015;26:327-36.

14. Feingold KR, Kazemi MR, Magra AL, McDonald CM, Chui LG, et al. ADRP/ADFP and Mal1 expression are increased in macrophages treated with TLR agonists. Atherosclerosis. 2010;209(1):81-8.

15. Kaushik S, Cuervo AM. Degradation of lipid droplet-associated proteins by chaperone mediated autophagy facilitates lipolysis. Nat Cell Biol. 2015;17(6):759-70.

16. Dice JF. Peptide sequences that target cytosolic proteins for lysosomal proteolysis. Trends Biochem Sci. 1990;15(8):305-9.

17. Chiang HL, Terlecky SR, Plant CP, Dice JF. A role for a 70-kilodalton heat shock protein in lysosomal degradation of intracellular proteins. Science. 1989;246(4928):382-5.

18. Cuervo AM, Dice JF. A receptor for the selective uptake and degradation of proteins by lysosomes. Science. 1996;273(5274):501-3.

19. Rodriguez-Navarro JA, Kaushika S, Koga H, Dall'Armi C, Shui G, et al. Inhibitory effect of dietary lipids on chaperone-mediated autophagy. Proc Natl Acad Sci U S A. 2012;109(12):E705-14.

20. Kaushik S, Cuervo AM. AMPK-dependent phosphorylation of lipid droplet protein PLIN2 triggers its degradation by CMA. Autophagy. 2016;12(2):432-8.

21. Hardie DG. AMPK: a key regulator of energy balance in the single cell and the whole organism. Int $J$ Obes (Lond). 2008;32:7-12.

22. Masuda $\mathrm{Y}$, Itabe $\mathrm{H}$, Odaki M, Hama K, Fujimoto $\mathrm{Y}$, et al. ADRP/adipophilin is degraded through the proteasome-dependent pathway during regression of lipid-storing cells. J Lipid Res. 2006;47(1):8798.

23. Xu G, Sztalryd C, Lu X, Tansey JT, Gan J, et al. Post-translational regulation of adipose differentiation-related protein by the ubiquitin/proteasome pathway. J Biol Chem. 2005;280(52):42841-7.

24. Xu G, Sztalryd C, Londos C. Degradation of perilipin is mediated through ubiquitination-proteasome pathway. Biochim Biophys Acta. 2006;1761(1):83-90.

25. Tomaru U, Takahashi S, Ishizu A, Miyatake Y, Gohda A, et al. Decreased proteasomal activity causes age-related phenotypes and promotes the development of metabolic abnormalities. Am J Pathol. 2012;180(3):963-72. 
26. Kola B, Grossman A, Korbonits M. The role of AMP-activated protein kinase in obesity. Front Horm Res. 2008;36:198-211.

27. Cordero MD, Williams MR, Ryffel B. AMP-activated protein kinase regulation of the NLRP3 inflammasome during aging. Trends Endocrinol Metab. 2018;29(1):8-17.

28. Pacifico L, Di Martino M, De Merulis A, Bezzi M, Osborn JF, et al. Left ventricular dysfunction in obese children and adolescents with nonalcoholic fatty liver disease. Hepatology. 2014;59(2):461-70.

29. Pacifico L, Anania C, Martino F, Cantisani V, Pascone R, et al. Functional and morphological vascular changes in pediatric nonalcoholic fatty liver disease. Hepatology. 2010;52(5):1643-51.

30. Pacifico L, Cantisani V, Ricci P, Osborn JF, Schiavo E, et al. Non-alcoholic fatty liver disease and carotid atherosclerosis in children. Pediatr Res. 2008;63(4):423-7.

31. Tanner JM. Growth and maturation during adolescence. Nutr Rev. 1981;39:43-55.

32. Cole TJ, Bellizzi MC, Flegal KM, Dietz WH. Establishing a standard definition for child ow and obesity worldwide: international survey. BMJ. 2000;320(7244):1240-3.

33. Matthews DR, Hosker JP, Rudenski AS, Naylor BA, Treacher DF, Turner RC. Homeostasis model assessment: insulin resistance and beta-cell function from fasting plasma glucose and insulin concentrations in man. Diabetologia. 1985;28:412-9.

34. Flynn JT, Kaelber DC, Baker-Smith CM, Blowey D, Carroll AE, et al. Clinical Practice Guideline for Screening and Management of High Blood Pressure in Children and Adolescents. Pediatrics: 2017; 140 (3): e20171904.

35. Cook S, Weitzman M, Auinger P, Nguyen M, Dietz WH. Prevalence of a metabolic syndrome phenotype in adolescents: findings from the third National Health and Nutrition Examination Survey, 1988-1994. Arch Pediatr Adolesc Med. 2003;157(8):821-7.

36. Shashaj B, Luciano R, Contoli B, Morino GS, Spreghini MR, et al. Reference ranges of HOMA-IR in normal-weight and obese young Caucasians. Acta Diabetol. 2016;53(2):251-60.

37. Alisi A, Manco M, Devito R, Piemonte F, Nobili V. Endotoxin and plasminogen activator inhibitor-1 serum levels associated with nonalcoholic steatohepatitis in children. J Pediatr Gastroenterol Nutr. 2010;50(6):645-9.

38. Niccoli G, D'Amario D, Borovac JA, Santangelo E, Scalone G, et al. Perilipin 2 levels are increased in patients with in-stent neoatherosclerosis: A clue to mechanisms of accelerated plaque formation after drug-eluting stent implantation. Int J Cardiol. 2018;258:55-8.

39. Son SH, Goo YH, Choi M, Saha PK, Oka K, et al. Enhanced atheroprotection and lesion remodelling by targeting the foam cell and increasing plasma cholesterol acceptors. Cardiovasc Res. 2016;109(2):294-304.

40. Sakamoto K, Sato Y, Shinka T, Sei M, Nomura I, et al. Proteasome subunits mRNA expressions correlate with male BMI: implications for a role in obesity. Obesity (Silver Spring). 2009;17(5):10449. 
41. Otoda T, Takamura T, Misu H, Ota T, Murata S, et al. Proteasome dysfunction mediates obesityinduced endoplasmic reticulum stress and insulin resistance in the liver. Diabetes. 2013;62:811-24.

\section{Figures}

A

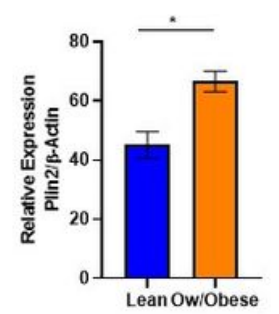

c

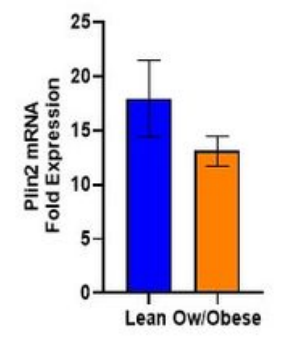

B
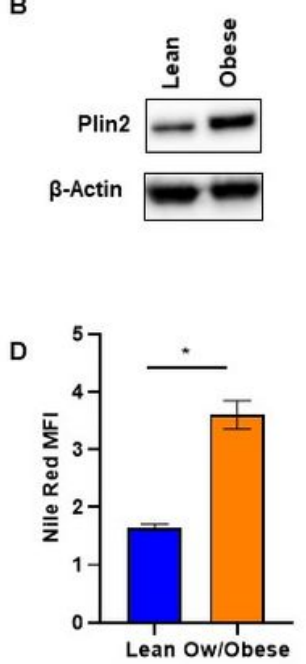

Figure 1

\section{Figure 1}

Plin2 protein expression is increased in ow/obese children. (A) Level of Plin2 in circulating monocytes of ow/obese and normal-weight children were analyzed by western blot and normalized for $\beta$-actin. Results are expressed as the mean (SEM). ${ }^{*} p=0,001$. (B) Representative blot of a pair normal-weight/obese is shown. (C) mRNA Plin2 fold expression in circulating monocytes of ow/obese and normal-weight children showed no significant difference. (D) Nile Red staining of monocytes showed a significant higher amount of intracellular lipids in ow/obese children as compared to normal weight controls. The results are expressed as the mean of MFI (SEM); $<<0.0001$. 

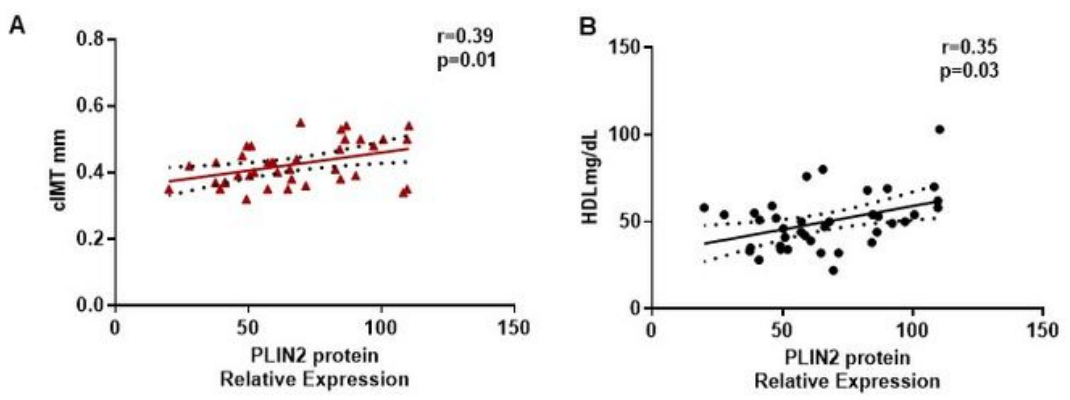

Figure 2

\section{Figure 2}

Plin2 expression level correlates with cIMT in obese children. (A) Univariate correlation analysis between monocyte Plin2 relative expression and cIMT in the ow/obese group. Following adjustments for covariates (age, sex, systolic blood pressure, diastolic blood pressure, body mass index z-score, hyperlipidemia, HOMA-index, lipopolysaccharides and soluble E-selectin level) Plin2 remained significantly associated with cIMT. (B) Correlation analysis between monocytes Plin2 relative expression and HDL-cholesterol in the obese group. 
A
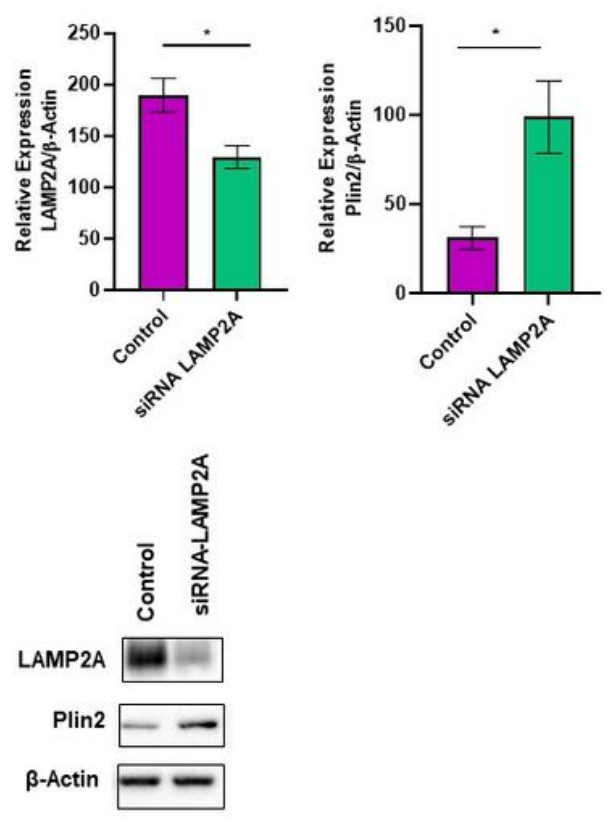

B
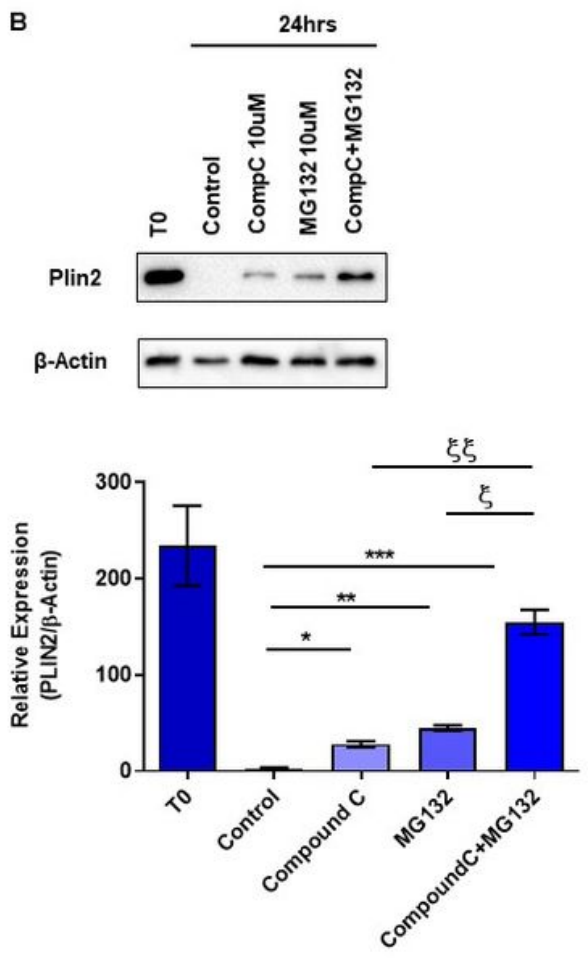

Figure 3

\section{Figure 3}

Both CMA and proteasome regulate Plin2 protein level in monocytes. (A) Primary monocytes were isolated from human donors and then transfected with gene specific siRNA duplexes for LAMP2A. After 48h, LAMP2A protein level was significantly reduced compared to control; $\mathrm{k}=0.03$. In parallel a significant fold increase of Plin2 protein level was observed; ${ }^{*} p=0.03$. The results are expressed as mean (SEM). Representative blot of a single experiment is shown in panel B. CTL represent cells transfected with scrambled siRNA. (B) Primary monocytes were isolated from human donors and treated with Compound C, MG132 and both of them for 24h. Compound C and MG132 treatments increased significantly Plin2 expression in monocytes and an additive effect was observed. The results are expressed as mean (SEM); ${ }^{*} \mathrm{p}=0.003 ;{ }^{* \star} \mathrm{p}=0.0004 ;{ }^{* \star} \mathrm{p}=0.0012 ; \xi \mathrm{p}=0.003 ; \xi \xi \mathrm{p}=0.003$. Representative blot of a single experiment is shown. T0 represent ex-vivo monocytes. CTL represent not treated cells. 

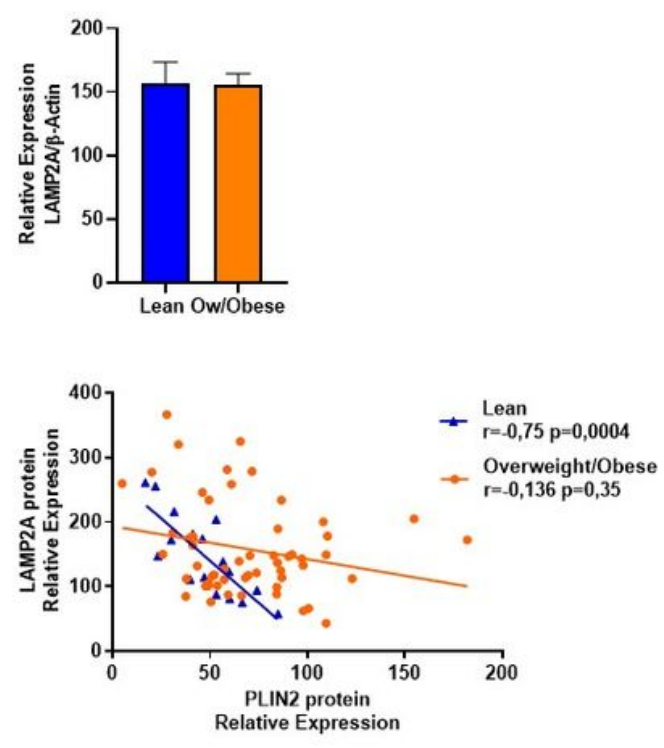

Figure 4

\section{Figure 4}

CMA evaluation in ow/obese and normal-weight children. Level of LAMP2A in circulating monocytes of ow/obese children and controls. The graphs depict densitometry analysis of LAMP2A protein normalized for $\beta$-actin. Results are expressed as mean (SEM); no significant difference was found in the two groups. Plin2 and LAMP2A protein level are inversely correlated in normal-weight but not in ow/obese children. 
A
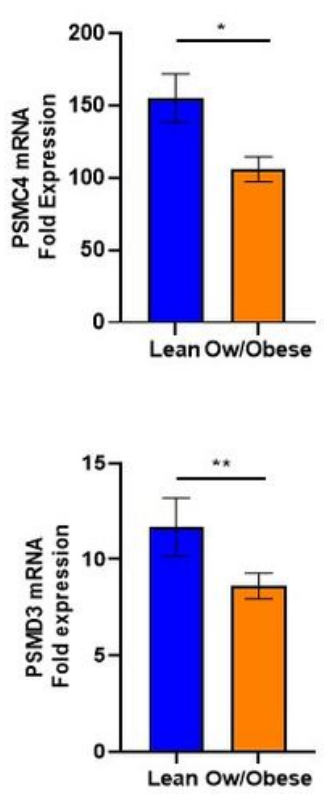

B
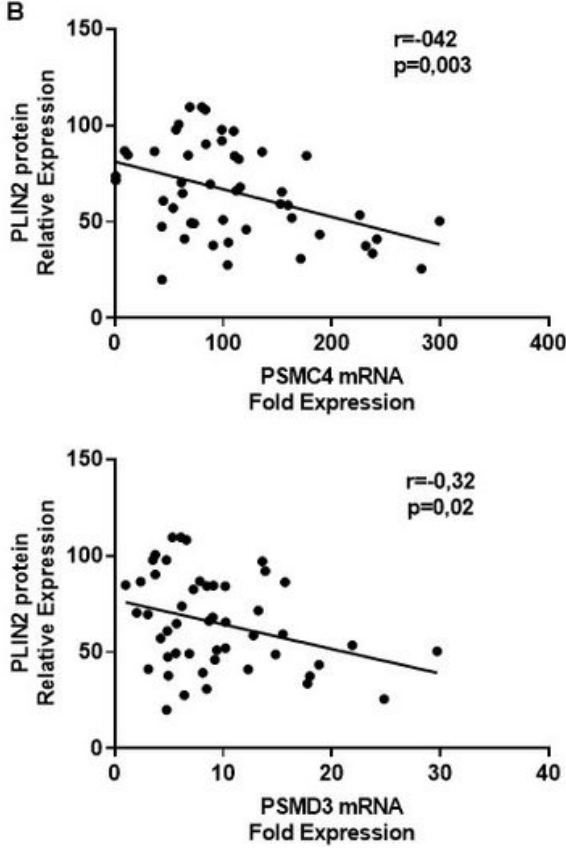

C

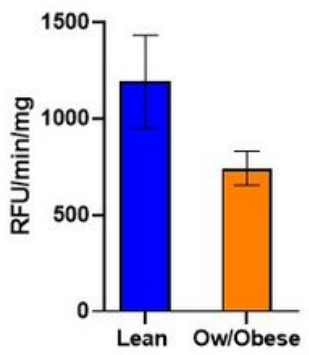

Figure 5

\section{Figure 5}

Proteasome evaluation in ow/obese and normal-weight children (A) PSMC4 and PSMD3 regulatory subunits mRNA expression was significantly decreased in ow/obese children. Results are expressed as mean (SEM); ${ }^{*}=0.006 ;{ }^{*} p=0.03$. (B) Significant negative correlation between PSMC4 and PSMD3 mRNA expression and Plin2 protein level in ow/obese children. (C) Proteasome activity was decreased in ow/obese compared to normal-weight children although the difference was not statistically significant. 


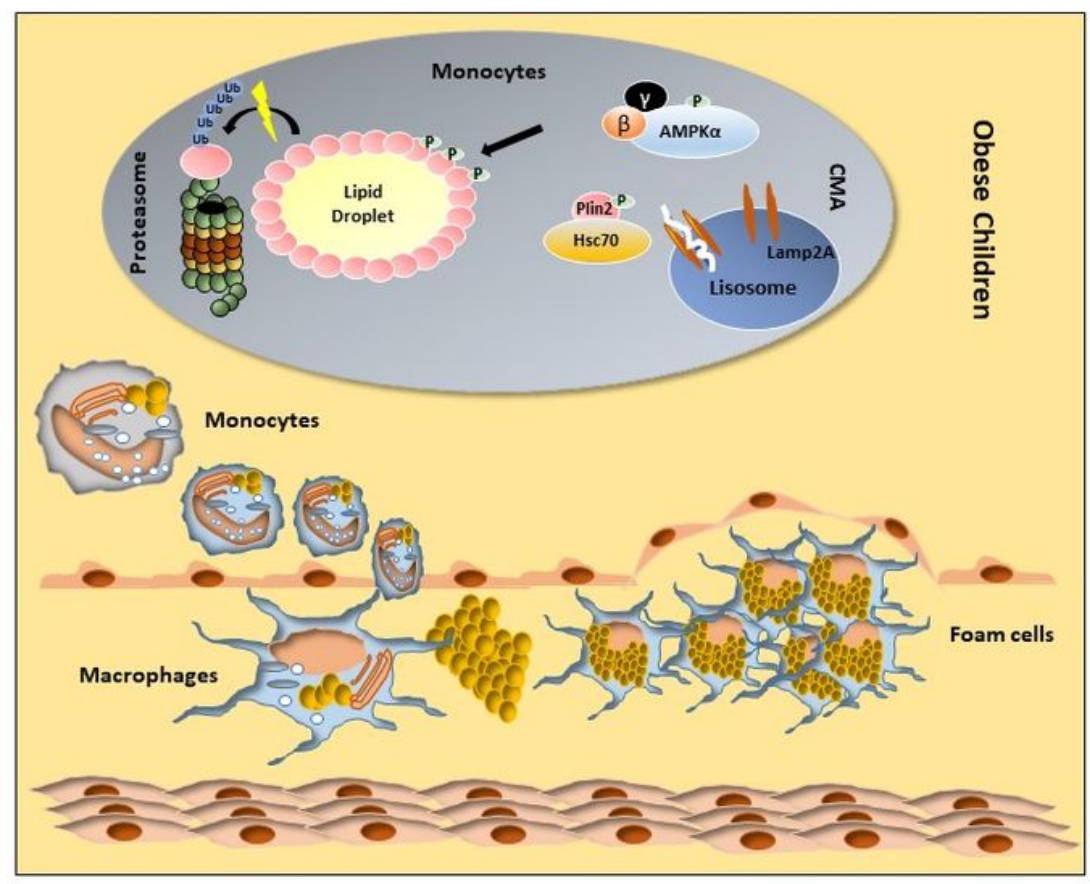

Figure 6

\section{Figure 6}

Schematic overview of proteasome dependent Plin2 dysregulation in monocytes of children with obesity. 